\title{
Perfil dos medicamentos fornecidos via processo judicial na assistência farmacêutica do município de Santa Rosa/RS.
}

\section{Profile of medicines supplied via judicial process in the pharmaceutical assistance in the city of Santa Rosa/RS.}

\section{Perfil de los medicamentos provistos vía proceso judicial en la asistencia farmacéutica del municipio de Santa Rosa/RS.}

\author{
Juliana Maronn UCKER ${ }^{1}$ \\ Karla Renata de OLIVEIRA ${ }^{2}$ \\ Vanessa Adelina Casali BANDEIRA ${ }^{3}$ \\ Vanessa Boeira FLORES ${ }^{4}$
}

RESUMO:Diante da crescente demanda por via judicial de acesso a medicamentos, é necessário conhecer as ações judiciais e os medicamentos solicitados, a fim de qualificar a assistência farmacêutica. O presente estudo objetiva identificar o perfil dos medicamentos fornecidos via processo judicial no município de Santa Rosa/RS. Trata-se de um estudo documental, transversal, analítico e descritivo. Foram incluídos no estudo os medicamentos dispensados no mês de dezembro de 2013 aos usuários ativos cadastrados para o fornecimento de medicamentos via judicial até dezembro daquele ano, no setor da Assistência Farmacêutica do município de Santa Rosa/RS. A amostra foi constituída por 451 usuários e os dados foram coletados através do sistema Administração de Medicamentos do Estado, no qual os usuários estão cadastrados. Foram

1 Farmacêutica, Universidade Regional do Noroeste do Estado do Rio Grande do Sul. Departamento de Ciências da Vida. Ijuí, RS, Brasil. E-mail: juliana.ucker@yahoo.com.br

2 Farmacêutica Mestre, docente do Departamento de Ciências da Saúde da Universidade Regional do Noroeste do Estado do Rio Grande do Sul. Departamento de Ciências da Vida. Ijuí, RS, Brasil. E-mail: karla@unijui.edu.br

3 Farmacêutica, Especialista em Saúde da Família pela Universidade Regional do Noroeste do Estado do Rio Grande do Sul e Fundação Municipal de Saúde de Santa Rosa. Ijuí, RS, Brasil. E-mail: vanessa.acbandeira@yahoo. com.br

4 Farmacêutica Coordenadora da Assistência Farmacêutica da Fundação Municipal de Saúde de Santa Rosa. Coordenação da Assistência Farmacêutica. Santa Rosa, RS, Brasil. E-mail: vafflores@yahoo.com.br 
dispensados 948 medicamentos no período do estudo, totalizando 390 especialidades farmacêuticas distintas. Duloxetina $60 \mathrm{mg}$ foi o medicamento mais dispensado. Os medicamentos classificados como fora das listas oficiais do Sistema Único de Saúde (SUS) foram os mais prevalentes, e dentre os incluídos em alguma lista, os do componente especializado foram os mais frequentes. Os resultados reforçam a importância da divulgação do elenco de medicamentos disponibilizados pelo SUS e dos Protocolos Clínicos e Diretrizes Terapêuticas tanto para usuários quanto para os prescritores que utilizam e atuam nos serviços públicos e privados de saúde do município, além da discussão para a inclusão de novos medicamentos nas listas oficiais do SUS.

Descritores: Assistência Farmacêutica, Direito à Saúde, Decisão Judicial, Sistema Único de Saúde.

ABSTRACT: By the increasing demand for judicial access to medicines, it is necessary to know the lawsuits and the required medicines in order to qualify the pharmaceutical care. This study aims to identify the profile of medicines supplied via judicial process in the city of Santa Rosa / RS. It is a documentary study, transversal, analytical and descriptive. The study included the medications dispensed in December 2013 to the registered active members for the supply of medicines via court until December of that year, in the section of the Pharmaceutical Care in the city of Santa Rosa / RS. The sample was consisted of 451 users and the data were collected through the State Drug Administration system in which users are registered. 948 drugs were dispensed during the study period, a total of 390 different pharmaceuticals. Duloxetine $60 \mathrm{mg}$ was the most dispensed medicine. Medicines classified as outside of the official lists of the Unified Health System (SUS) were the most prevalent, and among those included in any list, the specialized component were the most frequent. The results reinforce the importance of divulgation of the list of medications available through SUS and Clinical Protocols and Therapeutic Guidelines for both users and prescribers who use and work in public and private services of municipal health, beyond the discussion for the inclusion of new medicines on the official lists of SUS.

Keywords: Pharmaceutical Services, Right to Helth, Judicial Decisions, Unified Health System.

RESUMEN: Debido a la creciente demanda por la vía judicial de acceso a medicamentos, es necesario conocer las acciones judiciales y los medicamentos solicitados, con el fin de calificar la asistencia farmacéutica. El presente estudio tiene como objetivo identificar el perfil de los medicamentos provistos vía proceso judicial en el municipio de Santa Rosa/RS. Se trata de un estudio documental, transversal, analítico y descriptivo. Fueron incluidos en el estudio los medicamentos dispensados en el mes de Diciembre del 2013 a los usuarios activos registrados para la provisión de medicamentos vía judicial hasta Diciembre de dicho año, en el Sector de la Asistencia Farmacéutica del municipio de Santa Rosa/RS. La muestra fue constituida por 451 usuarios y los datos fueron recolectados por el Sistema de Administración de Medicamentos del Estado, en el cual los usuarios fueron registrados. Fueron dispensados 948 medicamentos en el 
periodo del estudio, totalizando 390 especialidades farmacéuticas distintas. Duloxetina $60 \mathrm{mg}$ fue el medicamento más dispensado. Los medicamentos clasificados como fuera de las listas oficiales del Sistema Único de Salud (SUS) fueron los más recurrentes, y los que estuvieron incluidos en alguna lista , los de componente especializado fueron los más frecuentes. Los resultados refuerzan la importancia de la divulgación del elenco de medicamentos disponibles por el SUS y de los Protocolos Clínicos y Directrices Terapéuticas tanto para los usuarios como para los prescriptores que utilizan y actúan en los servicios públicos y privados de la salud en el municipio, a parte de la discusión para la inclusión de nuevos medicamentos en las listas oficiales del SUS.

Descriptores: Asistencia Farmacéutica, Derecho a la Salud, Decisión Judicial, Sistema Único de Salud.

\section{INTRODUÇÃO}

O direito a assistência à saúde no Brasil foi inserido na Constituição Federal de 1988, que em seu artigo 196 reconhece a saúde como direito de todos e atribui ao Estado a responsabilidade por sua manutenção e execução ${ }^{1}$. Neste sentido, reformou-se a política de saúde pública brasileira, com a criação do Sistema Único de Saúde (SUS), implantado em 1990, por meio da Lei n 8.080, tendo como princípios base a universalização do acesso, a equidade na prestação de serviços e a integralidade da assistência terapêutica integral, inclusive farmacêutica, apresentando a importância do acesso aos medicamentos ${ }^{2}$.

O crescente processo de descentralização das ações e a criação do SUS contribuíram para a implantação de uma Política relacionada à Assistência Farmacêutica, que incorpora as prerrogativas estabelecidas pelo SUS.A partir daí, surge a Política Nacional de Medicamentos (PNM), que apresenta entre suas prioridades a adoção da Relação Nacional de Medicamentos Essenciais (RENAME) e a reorientação da Assistência Farmacêutica (AF), que se fundamenta na descentralização da gestão, na otimização e na eficácia do sistema de distribuição destes no setor público, no desenvolvimento de iniciativas que possibilitam a redução dos preços dos produtos, viabilizando, inclusive, o acesso da população aos produtos do setor privado ${ }^{3,4}$.

Tais diretrizes foram reafirmadas pela Política Nacional de Assistência Farmacêutica (PNAF) (2004) que objetiva ampliar o acesso, a promoção do Uso Racional de Medicamentos (URM) e aperfeiçoar o processo de descentralização das ações ${ }^{5}$.

Mais adiante, os Pactos pela Vida, em Defesa do SUS e de Gestão foram instituídos por meio da Portaria GM/MS n ${ }^{\circ} 399^{6}$. No Pacto de Gestão, o financiamento da AF foi definido como responsabilidade das três esferas de gestores do SUS, com foco na aquisição de medicamentos e insumos e a organização das ações de AF necessárias. A regulamentação da forma de transferência dos recursos financeiros federais foi instituída pela Portaria $n^{\circ} 204$ de 2007, estabelecendo o bloco de financiamento da AF, dividido em três componentes: o componente básico, estratégico e o especializado. Os medicamentos padronizados nestes componentes são avaliados no que se refere 
à eficiência e à efetividade, além da relação custo/benefício ${ }^{6,7,8}$.

Observa-se que houve avanços nas políticas e ações públicas de AF, no entanto, ainda há dificuldades de acesso da população aos medicamentos necessários à assistência integral à saúde. No Brasil, estimou-se que, em 2000, 70 milhões de pessoas não tinham acesso a medicamentos, o que correspondia a aproximadamente $41 \%$ da população brasileira ${ }^{9}$.

Uma das alternativas encontradas pelos usuários, para garantir o direito e o fornecimento do medicamento necessário, é a solicitação do seu medicamento através de processos judiciais. Segundo Leite et al. ${ }^{10}$, é um fenômeno que vem crescendo e tem sido considerado grande ameaça à sanidade financeira e administrativa do SUS. Romero ${ }^{4}$ salienta que essas decisões judiciais comprometem a dispensação regular, o atendimento de prioridades definidas e a implementação das políticas de AF aprovadas nos municípios, já que os gestores precisam remanejar recursos vultosos para atender situações isoladas.

As causas mais comuns de ingresso das ações judiciais, segundo levantamento do Conselho Nacional de Secretários de Saúde (CONASS) são a falta do medicamento ou a descontinuidade no tratamento por fornecimento irregular, recusa para efetuar o fornecimento pelo fato de a indicação estar em desacordo com os critérios estabelecidos pelos Protocolos Clínicos e Diretrizes Terapêuticas (PCDT) do Ministério da Saúde, ou ainda, medicamentos não padronizados, aqueles que não pertencem às listas públicas e os que não são ofertados por alguma das políticas publicas ou serviço do SUS ${ }^{11}$. Além disso, a crescente inovação tecnológica na indústria farmacêutica e o aumento de agravos à saúde principalmente de doenças crônicas podem ser determinantes ${ }^{12}$.

Através do fornecimento gratuito de medicamentos, o judiciário busca a garantia do direito à saúde e a promoção da universalização dos serviços. No entanto, a judicialização de medicamentos não promove o uso racional de medicamentos, além disso, a universalidade não significa que todos os medicamentos e tratamentos devem ser disponibilizados pelo SUS. Deve-se fornecer medicamentos efetivos e seguros, cuja relação risco-benefício seja favorável e comprovada por evidências científicas e com registro aprovado pela Agência Nacional de Vigilância Sanitária $(\text { ANVISA) })^{13}$.

Qualificar a gestão da AF é uma das ações fundamentais para enfrentar essa realidade, e para promover ações efetivas, é necessário buscar referenciais e conhecimento sobre as ações judiciais e os medicamentos solicitados. Nesse contexto, o objetivo do presente estudo foi identificar o perfil dos medicamentos fornecidos via processo judicial no município de Santa Rosa/RS.

\section{MÉTODOS}

Foi realizado um estudo documental, transversal, analítico e descritivo. Foram incluídos no estudo os medicamentos dispensados no mês de dezembro de 2013 aos usuários ativos cadastrados para o fornecimento de medicamentos via judicial até dezembro daquele ano, no setor da Assistência 
Farmacêutica do município de Santa Rosa/RS. Os medicamentos foram classificados por princípio ativo e dose.

Com a municipalização da saúde em Santa Rosa/RS, em 1995, foi criada a Fundação Municipal de Saúde de Santa Rosa (FUMSSAR), que abrange vários setores da Atenção Primária à Saúde, entre eles, o setor da Assistência Farmacêutica, que tem como prioridade assegurar o acesso da população à farmacoterapia de qualidade e contribuir para o uso racional de medicamentos. É coordenada por um profissional farmacêutico e assessorada tecnicamente pela Comissão de Farmácia e Terapêutica (CFT) que norteia as etapas do ciclo da AF. A CFT foi constituída através da publicação da Portaria $n^{0}$ 544/2009 ${ }^{14}$ e é composta por: um farmacêutico, dois médicos, um enfermeiro, e dois farmacêuticos Profissionais de Saúde Residentes e reúne-se em caráter ordinário mensalmente.

Atualmente o município conta com 17 Estratégias de Saúde da Família, sendo que cada unidade possui um dispensário de medicamentos, fornecendo aproximadamente 120 medicamentos para as doenças mais prevalentes. A farmácia especializada é centralizada e atende aproximadamente 4.500 usuários/mês fornecendo os medicamentos da lista de suporte da Relação Municipal de Medicamentos (REMUME) e do componente especializado através de pedidos administrativos e as demandas judiciais.

Os dados foram coletados através do sistema Administração de Medicamentos do Estado (AME), no qual os usuários estão cadastrados. As variáveis de interesse para a coleta de dados foram: medicamentos dispensados via judicial (princípio ativo e dose); idade e sexo dos usuários; doenças envolvidas e especialidade do médico prescritor.

Os medicamentos dispensados foram classificados no primeiro e quinto nível do sistema de classificação Anatomical Therapeutic Chemical $(A T C)^{15}$ e verificada sua inclusão nas listas oficiais do SUS, de acordo com principio ativo e dose, referentes ao ano de 2013, conforme Portaria MS no $1.554 / 2013$ e $n^{0} 1.555 / 2013$ e REMUME ${ }^{16,17}$.

No período do estudo também foram dispensados via judicial fórmulas, suplementos, dietas nutricionais e fita reativa para medição de glicemia, os quais foram excluídos do estudo.

Para a análise dos dados foi utilizado o programa estatístico Statistical Package for the Social Sciences (SPSS) para Windows versão 18.0. Para as variáveis categóricas utilizou-se a frequência relativa e absoluta, e para a variável quantitativa média e desvio padrão.

O protocolo de pesquisa foi aprovado pelo Comitê de Ética em Pesquisa da Universidade Regional do Noroeste do Estado do Rio Grande do Sul (UNIJUÍ), sob Parecer Consubstanciado ${ }^{\circ}$ 483.671/2013. 


\section{RESULTADOS}

Foram dispensados no período do estudo, 948 medicamentos, totalizando 390 especialidades farmacêuticas distintas, para 451 usuários.

Duloxetina $60 \mathrm{mg}$ foi o medicamento mais dispensado representando $2,6 \%$ do total dos medicamentos, seguido de formoterol $12 \mathrm{mcg}(2,3 \%)$; glicosamina $500 \mathrm{mg}+$ condroitina $400 \mathrm{mg}$ (2,1\%); citalopram 20mg (1,9\%) e insulina glargina $100 \mathrm{UI} / \mathrm{ml}$ (caneta) (1,5\%) (Tabela 1).

Tabela 1 - Distribuição dos 20 medicamentos mais dispensados via judicial no mês de dezembro de 2013 conforme presença em listas oficiais do SUS. Santa Rosa/RS.

\begin{tabular}{|c|c|c|}
\hline Medicamento & Presença em listas oficiais do SUS & Frequência \\
\hline Duloxetina $60 \mathrm{mg}$ & \multirow{11}{*}{ Fora de lista } & $2,6 \%$ \\
\hline Glicosamina $500 \mathrm{mg}+$ condroitina $400 \mathrm{mg}$ & & $2,1 \%$ \\
\hline Citalopram $20 \mathrm{mg}$ & & $1,9 \%$ \\
\hline Pantoprazol 40mg & & $1,5 \%$ \\
\hline Memantina $10 \mathrm{mg}$ & & $1,3 \%$ \\
\hline Glicosamina $1.5 \mathrm{~g}+$ condroitina $1,2 \mathrm{~g}$ & & $1,1 \%$ \\
\hline Paroxetina 20mg & & $0,9 \%$ \\
\hline Venlafaxina $75 \mathrm{mg}$ & & $0,8 \%$ \\
\hline Pregabalina $75 \mathrm{mg}$ & & $0,8 \%$ \\
\hline Rosuvastatina cálcica $10 \mathrm{mg}$ & & $0,8 \%$ \\
\hline TOTAL & & $13,8 \%$ \\
\hline Formoterol $12 \mathrm{mcg}$ & \multirow{5}{*}{ Componente especializado SES/RS* } & $2,3 \%$ \\
\hline Formoterol $12 \mathrm{mcg}+$ budesonida $400 \mathrm{mcg}$ & & $1,3 \%$ \\
\hline Beclometasona $400 \mathrm{mcg}$ & & $1,2 \%$ \\
\hline Quetiapina 100mg & & $0,8 \%$ \\
\hline TOTAL & & $5,6 \%$ \\
\hline Insulina Glargina $100 \mathrm{UI} / \mathrm{ml}$ & \multirow{4}{*}{ Componente especial SES/RS* } & $1,6 \%$ \\
\hline Sertralina $50 \mathrm{mg}$ & & $1,5 \%$ \\
\hline Travoprosta $0,04 \mathrm{mg} / \mathrm{ml}$ & & $0,8 \%$ \\
\hline TOTAL & & $3,9 \%$ \\
\hline Timolol $0,5 \%$ & \multirow{4}{*}{ REMUME } & $1,2 \%$ \\
\hline Omeprazol 20mg & & $0,9 \%$ \\
\hline Sinvastatina $20 \mathrm{mg}$ & & $0,8 \%$ \\
\hline TOTAL & & $2,9 \%$ \\
\hline
\end{tabular}

*Secretaria Estadual de Saúde do Estado do Rio Grande do Sul

Aclassificação dos medicamentos segundo o $1^{\circ}$ nível do código ATC indicou que os medicamentos mais dispensados via judicial no período do estudo atuam principalmente no Sistema Nervoso Central $(30,9 \%)$ e Sistema Cardiovascular $(15,3 \%)$ conforme mostra a Tabela 2. Além disso, a distribuição dos medicamentos dispensados via judicial quanto a presença em listas oficiais estão apresentados na Tabela 3 e sua classificação de acordo com ATC, na Tabela 4. 
Tabela 2 - Distribuição dos medicamentos dispensados via judicial no mês de dezembro de 2013 no primeiro nível da classificação ATC. Santa Rosa/RS.

\begin{tabular}{lcc}
\hline Primeiro nível ATC & Total & $\mathbf{\%}$ \\
\hline N- Sistema Nervoso & 292 & $30,9 \%$ \\
\hline C- Sistema Cardiovascular & 145 & $15,3 \%$ \\
\hline A- Aparelho digestivo e metabolismo & 106 & $11,2 \%$ \\
\hline R-Aparelho respiratório & 95 & $10 \%$ \\
\hline M- Sistema Músculo-Esquelético & 80 & $8,4 \%$ \\
\hline S- Órgãos dos Sentidos & 66 & $7,0 \%$ \\
\hline L- Agentes antineoplásicos e imunomoduladores & 43 & $4,5 \%$ \\
\hline B- Sangue érgãos hematopoiéticos & 41 & $4,3 \%$ \\
\hline H- Preparados hormonais sistêmicos & 23 & $2,4 \%$ \\
\hline G- Aparelho Geniturinário e hormônios sexuais & 16 & $1,7 \%$ \\
\hline J- Anti-infecciosos gerais para uso sistêmico & 12 & $1,3 \%$ \\
\hline D- Medicamentos e produtos dermatológicos & 3 & $0,3 \%$ \\
\hline P- Produtos antiparasitários, inseticidas e repelentes & $0,2 \%$ \\
\hline Medicamentos sem ATC & 2 & $2,5 \%$ \\
\hline Total de medicamentos & 24 & $100 \%$ \\
\hline
\end{tabular}

Tabela 3 - Distribuição dos medicamentos dispensados via judicial no mês de dezembro de 2013, segundo inclusão em listas oficiais do SUS. Santa Rosa/RS. Dezembro 2013

\begin{tabular}{lcc}
\hline Listas Oficiais & $\mathbf{\%}$ & $\mathbf{\%}$ \\
\hline Fora de Lista & 248 & 63,6 \\
\hline Componente Especializado- SES/RS & 67 & 7,2 \\
\hline REMUME & 31 & 7,9 \\
\hline Lista de suporte da Assistência Farmacêutica Municipal* & 9 & 4,1 \\
\hline Componente Básico- SES/RS & 16 & 4,1 \\
\hline Medicamentos Especiais- SES/RS & 16 & 0,8 \\
\hline Componente Estratégico- SES/RS & 3 & 100 \\
\hline Total & 390 & \\
\hline
\end{tabular}

*Medicamentos que compõe a REMUME dispensados somente na farmácia especializada

Em relação aos 451 usuários que receberam medicamento no mês de dezembro de 2013, a média

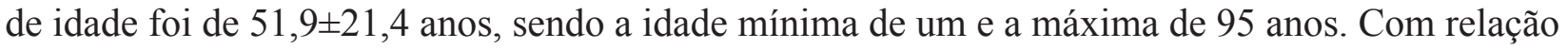
ao sexo, a maioria $(61,4 \%)$ são mulheres.

As doenças prevalentes, de acordo com a Classificação Internacional de Doenças (CID) foram: Transtornos mentais e comportamentais (F00-F99): 23,4\%; doenças do sistema osteomuscular e do tecido conjuntivo (M00-M99): 19,8\%; doenças do aparelho respiratório (J00-J99): 12,1\%; doenças endócrinas, nutricionais e metabólicas (E00-E90): 11\%; doenças do aparelho circulatório (I00-I99): 9,1\%; e doenças do sistema nervoso (G00-G99): 8,8\%, outras: 15,8\%

Quanto ao médico prescritor, foram identificados 155 médicos e 26 especialidades, com destaque para: Medicina da Família (12,2\%); Neurologia (8,3\%); Cardiologia, Psiquiatria, Oftalmologia (6,4\%); Ginecologia e Obstetrícia (5,1\%); Ortopedia e Traumatologia (4,5\%); Pneumologia (3,9\%); Gastroenterologia $(3,2 \%)$; outros $(56,4 \%)$ 
Tabela 4 - Medicamentos presentes nas listas oficiais do SUS dispensados via judicial no mês de dezembro de 2013 com classificação no nível 1 do código ATC. Santa Rosa/RS.

\begin{tabular}{|c|c|c|c|c|c|c|}
\hline Nível 1 código ATC & Especializado & REMUME & Básico & Especial & Estratégico & Total \\
\hline N- Sistema Nervoso & 28 & 6 & 1 & 5 & 1 & 41 \\
\hline C-Aparelho Cardiovascular & 6 & 15 & 6 & - & - & 27 \\
\hline $\begin{array}{l}\text { L- Agentes antineoplásicos e } \\
\text { moduladores }\end{array}$ & 13 & - & - & - & - & 13 \\
\hline A- Aparelho digestivo e & & & & & & \\
\hline Metabolismo & 2 & 4 & 4 & 2 & - & 12 \\
\hline R-Aparelho Respiratório & 8 & 3 & 2 & - & - & 12 \\
\hline $\begin{array}{l}\text { J-Anti-infecciosos gerais para } \\
\text { uso sistêmico }\end{array}$ & 3 & 3 & - & 1 & 2 & 9 \\
\hline S- Órgãos dos Sentidos & - & 1 & 2 & 5 & - & 8 \\
\hline $\begin{array}{l}\text { H- Preparados hormonais } \\
\text { sistêmicos }\end{array}$ & 3 & 4 & - & - & - & 7 \\
\hline $\begin{array}{l}\text { B- Sangue e órgãos } \\
\text { hematopoiéticos }\end{array}$ & 1 & 2 & - & 2 & - & 6 \\
\hline $\begin{array}{l}\text { M- Sistema músculo- } \\
\text { esquelético }\end{array}$ & 1 & 2 & 1 & - & - & 4 \\
\hline $\begin{array}{l}\text { G-Aparelho Genitourinário e } \\
\text { hormônios sexuais }\end{array}$ & 2 & - & - & - & - & 2 \\
\hline
\end{tabular}

\section{DISCUSSÃO}

Estudos revelam que as ações e atividades prestadas nos municípios brasileiros ainda são insuficientes para garantir o acesso e o uso racional de medicamentos pelo SUS ${ }^{18,19}$, o que leva à população buscar na via judicial a garantia do seu direito, como o identificado por Vieira e Zucchi ${ }^{20}$ em 36 municípios de cinco regiões do país identificaram que 38,9\% estavam cumprindo determinações judiciais para o fornecimento de medicamentos, principalmente da região centrosul do país.

Entre os medicamentos fornecidos por via judicial no município do presente estudo verificouse que mais da metade não fazem parte das listas oficiais do SUS (Tabela 3). Esse dado sugere o desconhecimento ou a pouca adesão dos prescritores a essas listas, pois ao considerar a RENAME contempla-se os tratamentos das doenças prevalentes no Brasil21 ${ }^{21}$ Reforça também a necessidade de garantir aos usuários que o processo de seleção dos medicamentos a serem disponibilizados pelo SUS e sua contínua atualização sejam baseadas nas melhores evidências científicas disponíveis e em avaliações farmacoeconômicas independentes ${ }^{13}$.

Resultados semelhantes foram verificados por Silva e Dalla Corte ${ }^{22}$ em estudo realizado na $12^{\mathrm{a}}$ Coordenadoria Regional de Saúde (CRS/RS) e por Macedo et al. ${ }^{23}$ em São Paulo, onde 63\% e $66,2 \%$ das medicações solicitadas judicialmente não estão contemplados nas listas oficiais do SUS, respectivamente.

Duloxetina $60 \mathrm{mg}$, o medicamento mais dispensado no período do estudo, não está incluído nas listas oficiais do SUS. Pertencente à classe dos Inibidores da Recaptação de Serotonina e 
Noradrenalina (IRSN), é indicado para tratamento da depressão, neuropatia diabética, transtorno de ansiedade generalizada, fibromialgia e dor muscular crônica ${ }^{24}$. Entretanto, de acordo com a Nota Técnica $N^{\circ}$ 45/2012 do Ministério da Saúde ${ }^{25}$, os diferentes antidepressivos apresentam eficácia semelhante para a maioria dos indivíduos deprimidos, variando em relação a efeitos colaterais e potencial de interação com outros medicamentos.

O SUS oferece as seguintes alternativas para o tratamento de transtorno depressivo: amitriptilina e imipramina, incluídos na classe dos antidepressivos tricíclicos (ADT), além de fluoxetina e sertralina, Inibidores Seletivos da Recaptação da Serotonina (ISRS) incluídos na REMUME de Santa Rosa/RS e no componente especial da SES/RS, respectivamente. Cabe salientar que, para o tratamento da depressão maior, segundo o Formulário Terapêutico Nacional (FTN) ${ }^{26}$, fluoxetina é significantemente mais bem tolerada que os ADTs. Além disso, citalopram, terceiro medicamento mais dispensado fora de lista, também pode ser substituído pelas alternativas contempladas nas listas citadas.

Entre os medicamentos supracitados, destaca-se que podem causar potenciais interações com outros medicamentos, sendo que dentre eles, a fluoxetina é o medicamento que apresenta maior número de interações ${ }^{24}$.

O CID correspondente a fibromialgia também foi relacionado à duloxetina. Neste caso, a amitriptilina, devido a capacidade de reduzir a dor e melhorar a capacidade funcional, também está recomendada para tratamento ${ }^{27}$.

Conforme a Lei $n^{0} 12.401$ de $2011^{28}$, a inclusão, exclusão ou a alteração pelo SUS de novos medicamentos nas listas oficiais, considera evidências científicas atualizadas sobre a eficácia, custo-efetividade e a segurança do medicamento aprovadas pelo órgão competente para o registro ou a autorização de uso. Uma das indicações da duloxetina se justifica pelo benefício singular que apresenta no tratamento da dor física associada à depressão ${ }^{25}$, o que provavelmente justifique os processos encaminhados. Porém, pela falta de novos estudos científicos relevantes comparando seu efeito com o de outros medicamentos, produtos contendo duloxetina ainda não foram incorporados às listas do SUS.

O segundo medicamento mais dispensado que não está presente nas listas oficiais do SUS, glicosamina 500mg + condroitina 400mg, é indicado para osteoartrite, osteoartrose, alterações degenerativas da articulação temporomandibular ${ }^{29}$. Médicos especialistas e o SUS ainda não apresentaram alternativas com a mesma eficácia e mecanismo de ação. No entanto, novos estudos deverão ser apresentados para que ocorra sua inclusão nas listas oficiais, o que provavelmente justifica que este medicamento seja um dos mais dispensados pela via judicial no município de Santa Rosa no período do estudo.

Considerando os CIDs prevalentes no presente estudo e a formação do prescritor, conclui-se que 
os medicamentos fora de lista foram prescritos por especialistas e que provavelmente não estejam vinculados à rede básica de saúde, e sim à rede complementar, o que reforça a necessidade de ampliar a divulgação e o estímulo à adesão de todos os profissionais que atuam no município aos PCDT e listas oficiais do SUS. Quanto ao grupo anatômico mais frequente do código ATC (Tabela 2) dados semelhantes foram encontrados nos estudos de Machado et al..$^{30}$ e Pepe et al. ${ }^{31}$, nos quais os medicamentos que atuam no sistema nervoso central e sistema cardiovascular são prevalentes. Estes dados indicam maior atenção da gestão da AF a estas doenças e medicamentos relacionados.

Em relação aos medicamentos dispensados no presente estudo que compõem as listas oficiais do SUS, a maioria pertence ao componente especializado (Tabela 3). Este componente tem como característica garantir a integralidade do tratamento medicamentoso, em nível ambulatorial, de agravos cujas abordagens terapêuticas estão estabelecidas nos PCDT, publicados pelo Ministério da Saúde. Estes protocolos representam um importante instrumento para a qualificação da assistência à saúde e tem como objetivo estabelecer os critérios de diagnóstico de cada doença, os tratamentos preconizados com as respectivas doses adequadas, monitoramento clínico em relação à efetividade do tratamento e a supervisão de possíveis efeitos adversos. Além disso, estabelecem mecanismos para a garantia da prescrição segura e eficaz ${ }^{16,32}$.

Formoterol $12 \mathrm{mcg}$, indicado para doenças respiratórias e presente no PCDT da asma ${ }^{32}$, foi o medicamento mais dispensado do componente especializado (tabela 1). Este resultado sugere o desabastecimento deste medicamento no estoque do Estado. Desta maneira, como o pedido administrativo não foi atendido, o usuário procura seu direito via judicial.

Em um estudo do CONASS, divulgado em 2004, as principais razões para o fornecimento via judicial de medicamentos do componente especializado em vários estados brasileiros são a falta dos medicamentos, o não cumprimento dos critérios dos PCDT e a solicitação de medicamentos não padronizados ${ }^{11}$.

Com o objetivo de esclarecer e regulamentar o acesso a medicamentos, a FUMSSAR em 2009, implantou a CFT no município. No mesmo ano, ocorreu a primeira reunião entre o judiciário, CFT e coordenador do setor da Assistência Farmacêutica da FUMSSAR, na qual, foi apresentada a estrutura da AF municipal e esclarecido ao judiciário sobre as competências das listas de medicamentos essenciais da União, Estado e Município. Além disso, estabeleceram-se fluxos de comunicação e critérios para o ajuizamento de ações, os quais incluem a emissão de certidão negativa, a prescrição do medicamento com uso da denominação comum brasileira, laudo médico com CID e justificativa para o uso. Atualmente são realizadas reuniões semestrais com o objetivo de reduzir o ajuizamento de ações referente a pedido de medicamentos e discutir as dificuldades frente aos fluxos das demandas judiciais.

$\mathrm{O}$ componente básico da $\mathrm{AF}$ se refere à aquisição de medicamentos e insumos no âmbito da 
atenção básica em saúde e àqueles relacionados a agravos e programas de saúde específicos, inseridos neste nível de atenção ${ }^{17}$. Estes medicamentos essenciais integram a RENAME, e a partir desta, a REMUME, que é elaborada para atender as necessidades específicas da população de cada município $^{26}$. A adoção de uma REMUME otimiza todas as etapas do ciclo da AF, e seu processo de elaboração deve ser feito de forma multiprofissional, objetivando maior adesão e consolidação.

A revisão da REMUME do município de Santa Rosa/RS é realizada anualmente pela CFT, e para a seleção dos medicamentos que serão mantidos, substituídos, incluídos ou excluídos, são utilizados, além da RENAME, critérios de farmacoeconomia, segurança, eficácia, comodidade posológica, e não adesão na prescrição por parte dos médicos. Os medicamentos presentes na REMUME estão organizados em listas considerando o local de dispensação. Assim, apresenta-se subdividida em: lista básica dos medicamentos dispensados nas Unidades de Saúde; lista de medicamentos para uso exclusivo das Unidades de Saúde; lista de suporte, com os medicamentos dispensados no setor de Assistência Farmacêutica, incluído entre estes, os medicamentos fitoterápicos e homeopáticos.

A maioria dos medicamentos dispensados judicialmente no período do estudo constantes na REMUME atuam no sistema cardiovascular (Tabela 4). Dados semelhantes foram encontrados no estudo de Barreto et al. ${ }^{33}$. Observou-se no presente estudo que estes processos judiciais muitas vezes ocorrem, não pelo desabastecimento, mas, devido algumas prescrições conterem todos os medicamentos de uso do paciente e quando apresentada ao advogado ou defensor, este inclui na ação todos os itens da receita, sem excluir os que compõem as listas oficiais do SUS. E assim, por falta de conhecimento destas listas, o judiciário, para garantir o tratamento que foi prescrito, defere todos os itens.

Diante disso, uma estratégia adotada a partir do ano de 2010 pelo município de Santa Rosa/ RS junto ao Poder Judiciário e Defensoria Pública, para evitar ações judiciais de medicamentos pertencentes à REMUME, é a emissão da certidão negativa, ou seja, somente serão ajuizadas ações de medicamentos fora das listas oficiais do SUS. Assim, é fundamental que sejam realizados estudos para verificar se as estratégias utilizadas estão sendo efetivas.

Observa-se que os medicamentos presentes nas listas oficiais do SUS no presente estudo $(36,4 \%)$ contribuem com uma parcela significativa das ações judiciais. No Distrito Federal, Honorato ${ }^{34}$ observou que a segunda causa mais frequente para o ingresso na via judicial (24,2\%) está relacionada à insuficiência no abastecimento de medicamentos das listas oficiais na Secretaria de Saúde do Estado. Segundo Machado et al..$^{30}$, falhas no gerenciamento da AF ou de alguma de suas etapas (programação, aquisição e distribuição) contribuem para solicitações destes medicamentos.

A etapa da seleção de medicamentos deve ser o eixo norteador de todas as políticas públicas envolvendo a AF, e deve se fundamentar nas melhores evidências científicas da literatura, considerar morbidades prevalentes, eficácia, efetividade, segurança e qualidade do fármaco, apresentações 
farmacêuticas que atendam as necessidades, comodidade posológica, custo e disponibilidade no mercado ${ }^{35}$. Diante disso, acredita-se que todas as listas oficiais do SUS estão baseadas nos critérios acima citados, por isso, estimular sua adesão por parte dos prescritores é a estratégia de maior impacto para evitar a judicialização. Atualmente, a inclusão de novos medicamentos nas listas também é baseada em demandas judiciais ${ }^{36}$.

A programação, outra etapa chave da AF, objetiva a garantia da disponibilidade dos medicamentos selecionados, nas quantidades adequadas e no tempo oportuno, para atender às necessidades da população ${ }^{35}$. Segundo Silva e Dalla Corte ${ }^{22}$, a judicialização desorganiza a programação e o fornecimento regular destes medicamentos à população e que além de interferir na questão orçamentária em nível municipal, tem gerado grandes dificuldades na gestão da AF do estado do Rio Grande do Sul.

De acordo com Araújo et al. ${ }^{37}$ para implantação da AF integral, que vai desde a pesquisa até a farmacovigilância, são necessários aperfeiçoamentos que possibilitem a avaliação de seu impacto na qualidade de vida do usuário, tendo como objetivo garantir o abastecimento e o acesso aos medicamentos, bem como o seu uso correto e efetivo.

Quando analisado o número de atendimentos mensais realizado no setor de Assistência Farmacêutica do município, observa-se que aproximadamente $10 \%$ são via judicial. Este é considerado um percentual baixo, quando comparado a outros municípios da $14^{\mathrm{a}} \mathrm{CRS}^{38}$. A judicialização não deveria se apresentar como um caminho para o acesso a medicamentos, porém, o fato da maioria dos medicamentos dispensados não estar presente nas listas oficiais do SUS, confirma que a judicialização é necessária para atender aos usuários em que os medicamentos do SUS não contempla, garantindo assim seu direito ao acesso.

Cabe destacar que a decisão judicial deveria ser baseada em parecer técnico emitido por uma CFT ou perito, uma vez que este seria baseado nas opções terapêuticas disponíveis no município e na eficácia e segurança assegurada por evidências clínicas disponíveis na literatura ${ }^{36}$. Sendo que, a dispensação de medicamentos pela via judicial que não apresentam registro na Anvisa, fora de sua indicação ou experimentais, podem expor o usuário a riscos, e por se tratar de tecnologias de alto custo, comprometer políticas públicas destinadas a coletividade ${ }^{39}$.

\section{CONSIDERAÇÕES FINAIS}

Constatou-se que os medicamentos mais dispensados por via judicial no município de Santa Rosa/RS, são os que atuam no sistema nervoso e sistema cardiovascular, sugere-se o planejamento e implantação de estratégias, tomando como ponto de partida os medicamentos não incluídos nas listas oficiais do SUS. Os resultados reforçam a importância da divulgação do elenco de medicamentos disponibilizados pelo SUS e dos PCDT tanto para usuários quanto para os prescritores que utilizam e atuam nos serviços público e privado de saúde do município, além da 
discussão para a inclusão de novos medicamentos nas listas oficiais do SUS.

O fornecimento de medicamentos via judicial presentes nas listas oficiais do SUS é necessário nos casos em que a via administrativa não garante o acesso, preservando o direito do usuário aos medicamentos previstos nas Políticas. Esse dado indica a importância da efetiva implementação do ciclo da $\mathrm{AF}$, principalmente em relação à seleção e programação e a informação sobre os medicamentos disponíveis, objetivando garantir o acesso e o uso racional de medicamentos.

Para enfrentar a realidade das ações judiciais e promover ações efetivas é fundamental que se conheça essas demandas e os medicamentos que estão sendo solicitados, bem como as doenças prevalentes na população atendida.

Os resultados deste estudo se constituem em subsídios para a reorientação dos serviços oferecidos pelo setor de Assistência Farmacêutica do município, e indicam a necessidade de ampliar e qualificar a comunicação entre o Poder Judiciário e profissionais da saúde, bem como o planejamento e implantação de estratégias visando à redução da judicialização da saúde, sem deixar a população desassistida.

\section{REFERÊNCIAS BIBLIOGRÁFICAS}

1. Brasil. Constituição Federal de 1988. Seção II da Saúde. Artigo, 196. Brasília: Presidente da República, 1988.

2. Brasil. Lei n. 8.080 de 19 de Setembro de 1990. Dispõe sobre as condições para a promoção, proteção e recuperação da saúde, a organização e o funcionamento dos serviços correspondentes e dá outras providências. Brasília, DF: Diário Oficial da União, 1990.

3. Brasil, Ministério da Saúde. Portaria MS n 3.916, de 30 de outubro de 1998. Aprova a Política Nacional de Medicamentos. Brasília, DF: Diário Oficial da União, 1998

4. Romero LC. Judicialização das políticas de assistência farmacêutica: o caso do distrito federal. Brasília: Consultoria Legislativa do Senado Federal, 2008.

5. Brasil, Ministério da Saúde. Resolução CNS n 338, de 6 de maio de 2004. Aprova a Política Nacional de Assistência Farmacêutica. Brasília: Ministério da Saúde, 2004.

6. Brasil, Ministério da Saúde. Portaria GM n 399 de 22 de fevereiro de 2006. Divulga o Pacto pela Saúde 2006 - Consolidação do SUS e aprova as Diretrizes Operacionais do referido Pacto. Brasília: Ministério da Saúde, 2006.

7. Brasil, Ministério da Saúde. Portaria GM/MS n. 204, de 29 de janeiro de 2007. Regulamenta o financiamento e a transferência dos recursos federais para as ações e os serviços 
de saúde, na forma de blocos de financiamento, com o respectivo monitoramento e controle. Brasília: Ministério da Saúde, 2007.

8. Chieffi AL, Barata RB. Judicialização da política pública de assistência farmacêutica e eqüidade. Cad Saúde Pública. 2009;25(8):1839-1849.

9. Vieira FS. Zucchi P. Distorções causadas pelas ações judiciais à política de medicamentos no Brasil. Revista de Saúde Pública. 2007;41(2):214 -222.

10. Leite SN, Pereira SMP, Silva P, Nascimento Jr. JM, Cordeiro BC, Veber AP. Ações Judiciais e Demandas Administrativas na Garantia do Direito de Acesso a Medicamentos em Florianópolis-SC. Rev. Direito Sanit. 2009;10(2):13-28.

11. Brasil, Conselho Nacional de Secretários de Saúde. Assistência Farmacêutica: medicamentos de dispensação em caráter excepcional. $1^{\text {a }}$ Ed. Brasília: CONASS, 2004

12. Brasil, Conselho Nacional de Secretários de Saúde. Nota técnica 19, de 15 de agosto de 2005. Brasília: CONASS, 2005.

13. Acurcio FA. Medicamentos: Políticas, Assistência Farmacêutica, Farmacoepidemiologia e Farmacoeconomia. Belo Horizonte: Coopmed; 2013.

14. Fundação Municipal de Saúde de Santa Rosa - FUMSSAR. Portaria $N^{o} 544$, de 4 de novembro de 2009. Nomeia a Comissão de Farmácia e Terapêutica. Santa Rosa-RS: 2009.

15. World Health Organization, Collaborating Centre for Drug Statistics Methodology. Anatomical Therapeutic Chemical ATC/DDD Index. Disponível em: http://www.whocc.no/atc ddd index/. Acessado em: 10 nov 2014.

16. Brasil, Ministério da Saúde. Portaria n $n^{o} 1554$ de 30 de julho de 2013. Dispõe sobre as regras de financiamento e execução do Componente Especializado da Assistência Farmacêutica no âmbito do Sistema Único de Saúde (SUS). Brasília: Ministério da Saúde; 2013.

17. Brasil, Ministério da Saúde. Portaria no .1555 de 30 de julho de 2013. Dispõe sobre as normas de financiamento e de execução do Componente Básico da Assistência Farmacêutica no âmbito do Sistema Único de Saúde (SUS). Brasília: Ministério da Saúde; 2013

18. Guerra Jr. AA, Acúrcio FA, Gomes CAP, Miralles M, Girardi SN, Carvalho CL, et al. Disponibilidade de medicamentos essenciais em duas regiões de Minas Gerais, Brasil. Rev Panam Salud Publica. 2004;15(3):168-75.

19. Fraga FNR. A utilização de um modelo lógico para a reorientação dos serviços farmacêuticos no âmbito municipal [Dissertação]. Porto Alegre (RS): Faculdade de Farmácia, 
Universidade Federal do Rio Grande do Sul; 2005.

20. Vieira FS, Zucci P. Gestão da Assistência Farmacêutica: Análise da situação de alguns municípios. Tempus actas de saúde colet., Brasília, 2014;8(4):11:29.

21. Brasil, Ministério da Saúde. Secretaria de Ciência, Tecnologia e Insumos Estratégicos, Departamento de Assistência Farmacêutica e Insumos Estratégicos. Relação Nacional de Medicamentos Essenciais: Rename 2013. 8.ed. Brasília: Ministério da Saúde; 2013.

22. Silva ARH, Dalla Corte E. Judicialização na Assistência Farmacêutica do Sistema Único de Saúde. Ciência em Movimento. 2011;27(1): 19-25.

23. Macedo EI, Lopes LC, Barberato-Filho, S. Análise técnica para a tomada de decisão do fornecimento de medicamentos pela via judicial. Rev Saúde Pública. 2011;45(4):706-13.

24. Dos Santos L, Torriani MS, Barros E. Medicamentos na prática da farmácia clínica. Porto Alegre: Artmed-2013.

25. Brasil, Ministério da Saúde. Nota Técnica N 45/2012 Duloxetina. Brasília, maio 2012.

1. 26. Brasil, Ministério da Saúde. Secretaria de Ciência, Tecnologia e Insumos Estratégicos, Departamento de Assistência Farmacêutica e Insumos Estratégicos. Formulário Terapêutico Nacional 2010: RENAME 2010. 2.ed. Brasília: Ministério da Saúde; 2010.

27. Heymann RE, Paiva EDS, Helfenstein Junior M, Pollak DF, Martinez JE, Provenza JR, et al. Consenso brasileiro de tratamento da fibromialgia. Rev Bras Reumatol. 2010;50(1):56-66.

28. Brasil. Lei $n^{\circ} 12.401$ de 2011. Altera a Lei $n^{\circ}$ 8.080, de 19 de setembro de 1990, para dispor sobre a assistência terapêutica e a incorporação de tecnologia em saúde no âmbito do Sistema Único de Saúde - SUS. Brasília: 2011

29. Machado E, Machado P, Cunali PA. Utilização de sulfato de condroitina e sulfato de glicosamina nas alterações degenerativas da ATM: uma revisão sistemática. Dental Press $J$ Orthod. 2012;17(4):1-5

30. Machado MAA, Acúrcio FA, Brandão CMR, Faleiros DR, Guerra Jr AA, Cherchiglia ML, et al. Judicialização do acesso a medicamentos no Estado de Minas Gerais, Brasil. Rev Saúde Pública. 2011;45(3):590-598.

31. Pepe VLE, Ventura M, Sant'Ana JMB, Figueiredo TA, Souza VR, Simas L, et al. Caracterização de demandas judiciais de fornecimento de medicamentos "essenciais" no Estado do Rio de Janeiro, Brasil. Cad Saúde Pública. 2010;26(3):461-71. 
32. Brasil, Ministério da Saúde, Secretaria de Atenção à Saúde. Protocolos clínicos e diretrizes terapêuticas. 2.ed.-Brasília: Ministério da Saúde, 2013.

33. Barreto JL, Pereira MT, Guimarães MCL, Penaforte TR, Formigli VLA. Perfil das demandas judiciais por medicamentos em municípios do estado da Bahia. Revista Baiana de Saúde Pública. 2013; 37(3):536-552.

34. Honorato, S. Judicialização da política de assistência farmacêutica: discussão sobre as causas de pedir no distrito federal [dissertação]. Brasília: Universidade de Brasília: 2014.

35. Marin N, Luiza VL, Castro CGSO, Santos SM, organizadores. Assistência farmacêutica para gerentes municipais. Rio de Janeiro: OPAS/OMS, 2003.

36. D’Espíndula TCAS. Judicialização da medicina no acesso a medicamentos: reflexões bioéticas. Rev. bioét. 2013; 121(3):438-47.

37. Araújo ALA, Ueta JM, Freitas O. Assistência farmacêutica como um modelo tecnológico em atenção primária à saúde. Rev. Ciênc. Farm. Básica Apl., 2005;26(2): 87-92.

38. Rio Grande do Sul. $14^{\text {a }}$ Coordenadoria Regional da Saúde. Relatórios do sistema AME: 2014 .

39. Santos AO, Delduque MC, Mendonça AVM. Os discursos na Audiência Pública da Saúde e seu impacto nas decisões do Supremo Tribunal Federal: uma análise à luz da teoria dos sistemas sociais. Saúde Soc. São Paulo. 2015;24(supl.1):184-192.

Artigo apresentado em 09-09-15

Artigo aprovado em 12-02-16

Artigo publicado no sistema em 30-03-16 